\title{
A conjecture of Stanley on alternating permutations
}

\author{
Robin Chapman and Lauren K. Williams \\ Department of Mathematics, University of Bristol, Bristol, BS8 1TW, UK \\ Department of Mathematics, Harvard University, Cambridge, MA 02138 \\ marjc@bristol.ac.uk \\ lauren@math . harvard.edu
}

Submitted: Apr 3, 2007; Accepted: Jul 18,2007; Published: Jul 26, 2007

Mathematics Subject Classification: 05Axx

\begin{abstract}
We give two simple proofs of a conjecture of Richard Stanley concerning the equidistribution of derangements and alternating permutations with the maximal number of fixed points.
\end{abstract}

\section{Introduction}

We write $[n]=\{1, \ldots, n\}$ and $S_{n}$ for the set of permutations of $[n]$. A permutation is alternating if $a_{1}>a_{2}<a_{3}>a_{4}<\ldots$ Similarly, define $w$ to be reverse alternating if $a_{1}<a_{2}>a_{3}<a_{4}>\ldots$

In [2], Richard Stanley used the theory of symmetric functions to enumerate various classes of alternating permutations $w$ of $\{1,2, \ldots n\}$. One class that he considered were alternating permutations $w$ with a specified number of fixed points.

Write $d_{k}(n)$ (respectively, $d_{k}^{*}(n)$ ) for the number of alternating (respectively, reverse alternating) permutations in $S_{n}$ with $k$ fixed points.

As observed in [2], it is not hard to see that

$$
\begin{aligned}
& \max \left\{k: d_{k}(n) \neq 0\right\}=\lceil n / 2\rceil, n \geq 4 \\
& \max \left\{k: d_{k}^{*}(n) \neq 0\right\}=\lceil(n+1) / 2\rceil, n \geq 5 .
\end{aligned}
$$

Stanley conjectured the following in [2].

Conjecture 1. [2, Conjecture 6.3] Let $D_{n}$ denote the number of derangements (permutations without fixed points) in $S_{n}$. Then

$$
\begin{aligned}
d_{\lceil n / 2\rceil}(n) & =D_{\lfloor n / 2\rfloor}, n \geq 4 \\
d_{\lceil(n+1) / 2\rceil}^{*}(n) & =D_{\lfloor(n-1) / 2\rfloor}, n \geq 5 .
\end{aligned}
$$



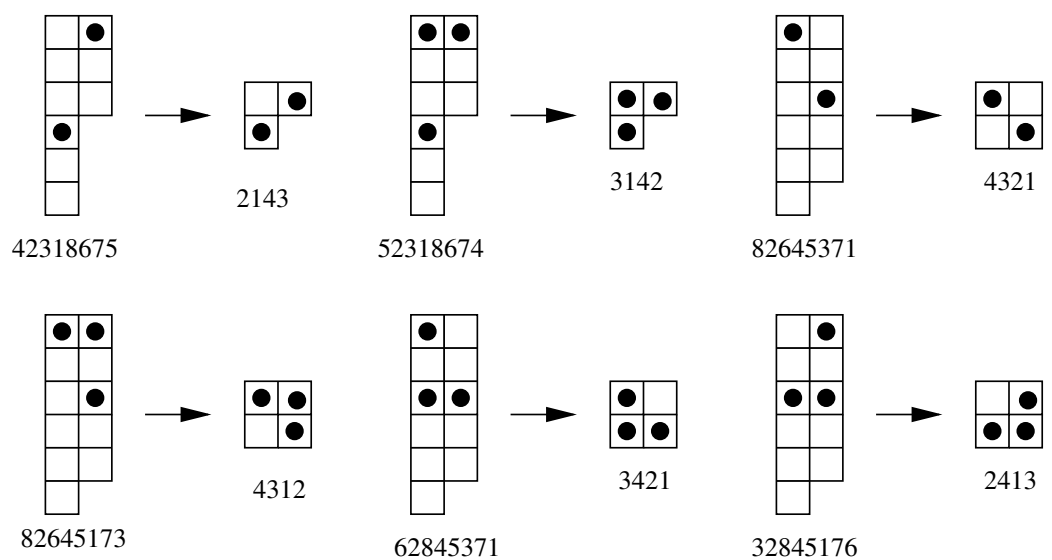

82645371
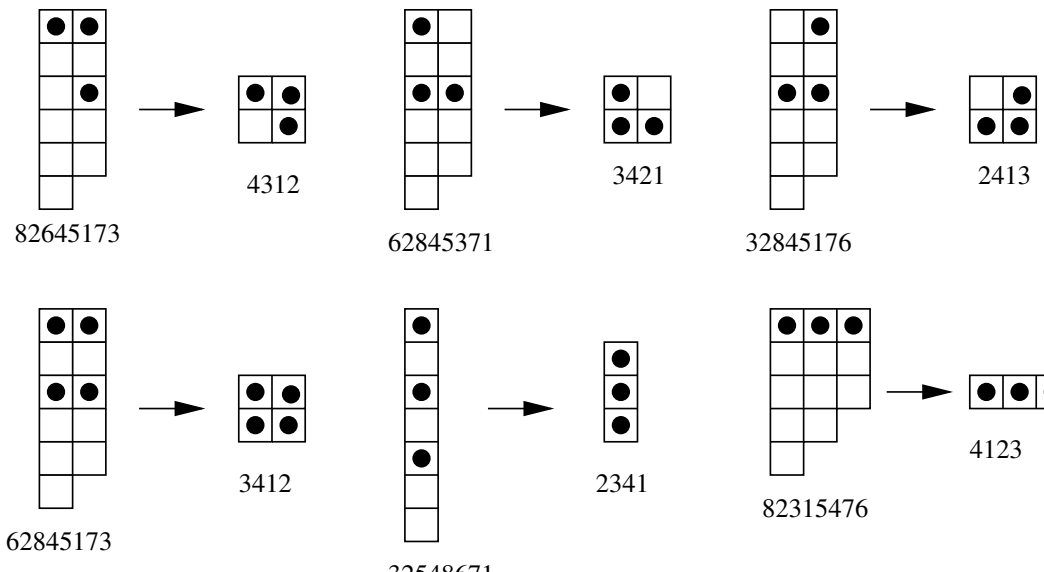

Figure 1: The bijection $\Psi$ for $n=8$

In this note we will give two proofs of his conjecture relating the number of derangements to the number of alternating permutations with the maximal number of fixed points. Both proofs use the same bijection $\Psi$. The first proof works directly with permutations and shows that $\Psi$ is injective and surjective. The second proof works with permutation tableaux, certain tableaux which are naturally in bijection with permutations, and explicitly constructs the inverse to $\Psi$. The bijection (for alternating permutations) is illustrated in Figure 1, in terms of both permutations and permutation tableaux.

\section{The first proof}

As we will show subsequently, the main case that one needs to consider concerns alternating permutations on an even number of letters.

Theorem 2. For each nonnegative integer $m$

$$
d_{m}(2 m)=D_{m} .
$$

Proof. Let $\mathcal{A}_{m}$ denote the set of alternating permutations of $[2 m]$ with exactly $m$ fixed points and let $\mathcal{D}_{m}$ denote the set of derangements of $[m]$. We shall define an explicit bijection $\Psi: \mathcal{A}_{m} \rightarrow \mathcal{D}_{m}$.

Let $\pi \in S_{2 m}$ be an alternating permutation. We partition the set $[2 m]$ into $m$ twoelement subsets $I_{1}, \ldots, I_{m}$ by setting $I_{j}=\{2 j-1,2 j\}$. As $\pi$ is alternating, $\pi(2 j-1)>$ $\pi(2 j)$ and so $2 j-1$ and $2 j$ cannot both be fixed points of $\pi$. Hence $\pi$ has at most $m$ 
fixed points, and if $\pi \in \mathcal{A}_{m}$ then $\pi$ fixes exactly one point in each $I_{j}$ and moves the other point. Write $I_{j}=\left\{a_{j}, b_{j}\right\}$ where $\pi\left(a_{j}\right)=a_{j}$ and $\pi\left(b_{j}\right) \neq b_{j}$. Thus $\pi$ permutes the $b_{j}$ with no fixed points, that is there is a unique derangement $\sigma \in \mathcal{D}_{m}$ with $\pi\left(b_{j}\right)=b_{\sigma(j)}$. Set $\Psi(\pi)=\sigma$.

To prove that $\Psi$ is a bijection we prove that it is injective and surjective. Suppose $\pi \in \mathcal{A}_{m}$ and $\Psi(\pi)=\sigma$. If $\sigma(j)>j$ then

$$
\pi\left(b_{j}\right)=b_{\sigma(j)} \geq 2 \sigma(j)-1>2 j \geq a_{j}=\pi\left(a_{j}\right) .
$$

As $\pi$ is decreasing on $I_{j}$ then $b_{j}=2 j-1$ and $a_{j}=2 j$. Similarly if $\sigma(j)<j$ then $a_{j}=2 j-1$ and $b_{j}=2 j$. Hence $\sigma$ determines the $a_{j}$ and $b_{j}$. Then as $\pi\left(a_{j}\right)=a_{j}$ and $\pi\left(b_{j}\right)=b_{\sigma(j)}$ then $\sigma$ also determines $\pi$. Thus $\Psi$ is injective.

To prove $\Psi$ is surjective take $\sigma \in \mathcal{D}_{m}$. Define

$$
\left(a_{j}, b_{j}\right)= \begin{cases}(2 j-1,2 j) & \text { if } \sigma(j)<j \\ (2 j, 2 j-1) & \text { if } \sigma(j)>j\end{cases}
$$

Then each element of $[m]$ is labelled as either an $a_{j}$ or a $b_{j}$. Define $\pi \in S_{2 m}$ by $\pi\left(a_{j}\right)=a_{j}$ and $\pi\left(b_{j}\right)=b_{\sigma(j)}$. As $\sigma$ is a derangement then $\pi$ has exactly $m$ fixed points. We claim that $\pi$ is alternating. First of all if $\sigma(j)<j$ then

$$
\pi(2 j)=\pi\left(b_{j}\right)=b_{\sigma(j)} \leq 2 \sigma(j)<2 j-1=\pi(2 j-1) .
$$

A similar argument works also when $\sigma(j)>j$. We also need to prove that $\pi(2 j)<$ $\pi(2 j+1)$ for $1 \leq j \leq m-1$. Now as $\pi(2 j)<\pi(2 j-1)$ and one of $\pi(2 j)=2 j$ and $\pi(2 j-1)=2 j-1$ holds then $\pi(2 j) \leq 2 j$. Similarly $\pi(2 j+1) \geq 2 j+1$. Hence $\pi(2 j)<\pi(2 j+1)$ and $\pi$ is alternating. It follows that $\pi \in \mathcal{A}_{m}$ and clearly $\Psi(\pi)=\sigma$. Hence $\Psi$ is a bijection and this concludes the proof.

Regarding a permutation as the list of its values we can describe the action of $\Psi$ in a simple way. Take a permutation $\pi \in \mathcal{A}_{m}$ and first delete all fixed points. Then replace each number $k$ in the remaining list by $\lceil k / 2\rceil$. For example take $\pi=52318674$. Deleting its fixed points gives 5184, and halving and rounding up each entry gives $\Psi(\pi)=3142$.

The remaining cases are simple consequences of this.

Theorem 3. For each positive integer $m$

$$
d_{m+1}^{*}(2 m)=D_{m-1}
$$

and

$$
d_{m}(2 m-1)=d_{m}^{*}(2 m-1)=D_{m-1} .
$$

Proof. A reverse alternating permutation of $[2 m]$ having $m+1$ fixed points must fix 1 and $2 m$. Also it restricts to an alternating permutation of $\{2, \ldots, 2 m-1\}$ with $m-1$ fixed points and so $d_{m+1}^{*}(2 m)=d_{m-1}(2 m-2)=D_{m-1}$. Conjugating with the reversal permutation $\rho: j \mapsto 2 m-j$ of [2m-1] interchanges alternating permutations in $S_{2 m-1}$ with $m$ fixed points with reverse alternating permutations in $S_{2 m-1}$ with $m$ fixed points. Hence $d_{m}(2 m-1)=d_{m}^{*}(2 m-1)$. Also an alternating permutation of $[2 m-1]$ with $m$ fixed points must fix $2 m-1$ and so restricts to an alternating permutation of $[2 m-2]$ with $m-1$ fixed points. Hence $d_{m}(2 m-1)=d_{m-1}(2 m-2)=D_{m-1}$. 


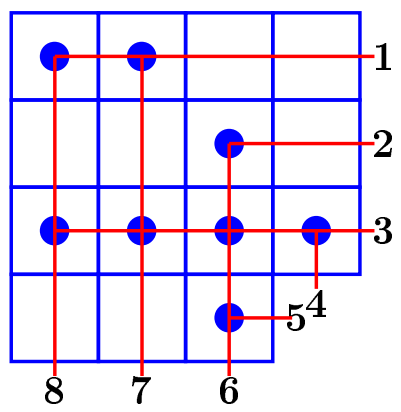

Figure 2: The diagram of a tableau.

\section{Permutation Tableaux}

A partition $\lambda=\left(\lambda_{1}, \ldots, \lambda_{k}\right)$ is a weakly decreasing sequence of nonnegative integers. For a partition $\lambda$, the Young diagram $Y_{\lambda}$ of shape $\lambda$ is a left-justified diagram of boxes, with $\lambda_{i}$ boxes in the $i$-th row.

A permutation tableau $\mathcal{T}[1,3]$ is a partition $\lambda$ together with a filling of each box of $Y_{\lambda}$ with either a (black) dot or nothing such that the following holds:

1. Each column of $Y_{\lambda}$ contains at least one dot.

2. There is no empty box which has a dot above it in the same column and a dot to its left in the same row.

Figure 2 gives an example of a permutation tableau.

We now recall the bijection $\Phi$ from permutation tableaux to permutations. More precisely, $\Phi$ is a bijection from the set of permutation tableaux with $k$ rows and $n-k$ columns to permutations in the symmetric group $\mathcal{S}_{n}$ with $k$ weak excedances. Here, a weak excedance of a permutation $\pi$ is a value $\pi(i)$ such that $\pi(i) \geq i$. If we define the semiperimeter of a tableau to be the number of columns plus the number of rows, then $\Phi$ maps the set of permutation tableaux of semiperimeter $n$ to permutations in $\mathcal{S}_{n}$.

We define the diagram $D(\mathcal{T})$ associated with $\mathcal{T}$ as follows. Label the edges of the northeast border of the Young diagram with the numbers from 1 to $n$, as in Figure 2 . From each black dot $v$, draw an edge to the east and an edge to the south; each such edge should connect $v$ to either a closest vertex in the same row or column, or to one of the labels from 1 to $n$. The resulting picture is the diagram $D(\mathcal{T})$.

We now define the permutation $\pi=\Phi(\mathcal{T})$ via the following procedure. For each $i \in\{1, \ldots, n\}$, find the corresponding position on $D(\mathcal{T})$ which is labeled by $i$. If the label $i$ is on a vertical step of $P$, start from this position and travel straight west as far as possible on edges of $D(\mathcal{T})$. Then, take a "zig-zag" path southeast, by traveling on edges of $D(\mathcal{T})$ south and east and turning at each opportunity (i.e. at each new vertex). This path will terminate at some label $j \geq i$, and we let $\pi(i)=j$. If $i$ is not connected to any edge then we set $\pi(i)=i$. Similarly, if the label $i$ is on a horizontal step of $P$, start from this position and travel north as far as possible on edges of $D(\mathcal{T})$. Then, as before, take 


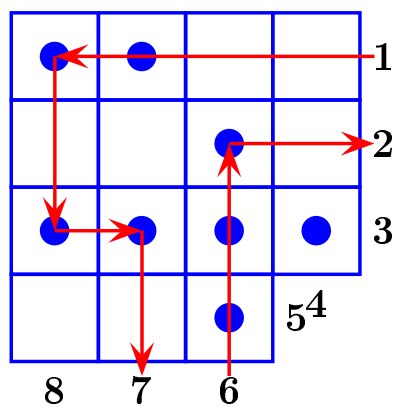

Figure 3: The paths taken by 1 and $6: \pi(1)=7, \pi(6)=2$.

a zig-zag path south-east, by traveling on edges of $D(\mathcal{T})$ east and south, and turning at each opportunity. This path will terminate at some label $j<i$, and we let $\pi(i)=j$.

See Figure 3 for a picture of the path taken by $i$.

Example 4. If $\mathcal{T}$ is the permutation tableau whose diagram is given in Figures 2 and 3, then $\Phi(\mathcal{T})=74836215$

The following lemma is clear from the construction above.

Lemma 5. [3] The positions of the weak excedances of $\pi=\Phi(\mathcal{T})$ are precisely the labels on the vertical edges of $P$. The positions of non-excedances of $\pi$ are precisely the labels on the horizontal edges of $P$. Furthermore, in $\Phi(\mathcal{T})$, the letter $i$ is a fixed point if and only if the row in $\mathcal{T}$ whose right hand edge is labeled by $i$ does not contain any dots (is "empty").

\section{The second proof}

Let $A T_{n}(2 n)$ denote the set of permutation tableaux corresponding to alternating permutations in $S_{2 n}$ with $n$ fixed points, and let $D T(n)$ denote the set of permutation tableaux corresponding to derangements in $S_{n}$. We will describe $\Psi$ on the level of tableaux and prove that it is a bijection between $A T_{n}(2 n)$ and $D T(n)$.

By Lemma 5 , the set $D T(n)$ consists of permutation tableaux with semiperimeter $n$ such that no row is empty (i.e. each row contains at least one black dot). We define $\Psi$ on the set $A T_{n}(2 n)$ : it acts on a tableau $\mathcal{T}$ by deleting all empty rows. See Figure 1. Clearly $\Psi(\mathcal{T}) \in D T(n)$ for some $n$.

We now define a map $\Theta$ on $D T(n)$ (illustrated in Figure 4), which will be the inverse of $\Psi$, and acts by inserting precisely $n$ empty rows into the tableau $\mathcal{T}$. More specifically, if $\mathcal{T} \in D T(n)$ with partition shape $\left(\lambda_{1}, \lambda_{2}, \ldots, \lambda_{k}\right)$, then $\Theta(\mathcal{T})$ is the tableau which results after performing the following algorithm:

- For every $i$ such that $1 \leq i \leq k-1$, insert $\lambda_{i}-\lambda_{i+1}+1$ empty rows between the $i$ th and $(i+1)$ st rows of $\mathcal{T}$, whose rows lengths are $\left(\lambda_{i}, \lambda_{i}, \lambda_{i}-1, \lambda_{i}-2, \lambda_{i}-3, \ldots, \lambda_{i+1}+1\right)$. 
- Insert $\lambda_{k}+1$ empty rows after the $k$ th (last) row of $\mathcal{T}$, whose row lengths are $\left(\lambda_{k}, \lambda_{k}, \lambda_{k}-1, \lambda_{k}-2, \ldots, 2,1\right)$.

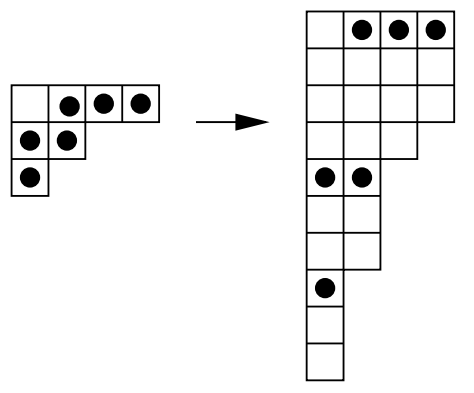

Figure 4:

The following lemma is obvious.

Lemma 6. Let $\pi$ be an alternating permutation. If $k$ consecutive entries are fixed points, then $k \leq 2$. Furthermore, if two consecutive entries $a_{i}$ and $a_{i+1}$ are fixed points, then $a_{i}<a_{i+1}$ and $i$ is even.

We now give a second proof of Theorem 2 .

Proof. We need to prove that $\Psi$ gives a bijection from $A T_{n}(2 n)$ to $D T(n)$, whose inverse is $\Theta$. It is obvious that $\Theta$ is an injection, that $\Psi \circ \Theta$ is the identity, and that if $\mathcal{T}$ is a tableau corresponding to a derangement then $\Theta(\mathcal{T})$ has $n$ fixed points. However, we need to show that $\Theta(\mathcal{T})$ is alternating and that $\Psi$ is an injection.

We will first show that $\Psi$ is an injection. Suppose we are trying to insert $n$ empty rows into $\mathcal{T}$, so as to get a tableau $\mathcal{T}^{\prime}$ corresponding to an alternating permutation $\pi$. Clearly the first row of the resulting tableau $\mathcal{T}^{\prime}$ cannot be empty, because then $\pi(1)=1$ violates the requirement than $\pi(1)>\pi(2)$. Additionally, by Lemma 6 , we cannot have three consecutive empty rows of the same length.

Consider two consecutive rows in $\mathcal{T}$ which have lengths $t \geq s$. Let us try to maximize the number of empty rows that we can insert between these rows, such that the resulting permutation $\pi$ will be alternating. Suppose these rows have lengths $r_{1}, r_{2}, \ldots, r_{m}$, with $t \geq r_{1} \geq r_{2} \geq \cdots \geq r_{m} \geq s$. As before, there cannot be three consecutive $r_{i}$ 's which are equal. Furthermore, note that after the rows of length $t$, there cannot be two empty rows of the same length. Indeed, if there were two such rows (say with vertical edge labels $j$ and $j+1$ ) then since $j-1$ is the label of a horizontal step of the tableau - i.e. the position of a nonexcedance - we would have $\pi(j-1)<j-1, \pi(j)=j$, and $\pi(j)=j+1$. This violates the requirement that $\pi$ be alternating.

So the best we could hope for is to have empty rows of lengths $t, t, t-1, t-2, \ldots, s+1, s$. But in fact we cannot have an empty row of length $s$. If we did, then if $j$ is the label of the vertical step of this row, then we would have $\pi(j-1)<j-1$ (since $j-1$ would be the label of a horizontal step, hence a position of a nonexcedance). Additionally, we would 
have $\pi(j)=j$ and $\pi(j+1) \geq j+1$, and these three entries would violate the requirement that $\pi$ be alternating.

Therefore if we want to insert $n$ empty rows into $\mathcal{T}$ such that the resulting permutation is alternating, then our only choice is to use the algorithm described in the definition of $\Theta$. Therefore $\Psi$ is an injection.

Finally it remains to show that the permutation corresponding to $\Theta(\mathcal{T})$ is alternating. Consider two consecutive rows of lengths $\lambda_{i}$ and $\lambda_{i+1}$ in $\mathcal{T}$, and suppose that the vertical column labels corresponding to these rows are $j$ and $k$. We will show that the entries of the corresponding permutation from positions $j$ to $k$ are alternating. Since the row of length $\lambda_{i}$ has at least one dot, we have $\pi(j)>j$. Since $j+1$ and $j+2$ label the vertical steps of empty rows, we have $\pi(j+1)=j+1$ and $\pi(j+2)=j+2$. Since $j+3$ labels a horizontal step, $\pi(j+3)<j+3$ (and hence less than $j+2$ since $\pi(j+2)=j+2$ ). Since $j+4$ labels a vertical step, $\pi(j+4)=j+4$. Continuing in this fashion, we finally check that $\pi(k-2)=k-2, \pi(k-1)<k-1$ (hence less than $k-2$ ), and $\pi(k)>k$. Clearly $\pi$

is alternating between the positions $j$ and $k$, and since the same argument can be applied to the remaining positions of $\pi$, we are done.

\section{References}

[1] A. Postnikov, Total positivity, Grassmannians, and networks. math.CO/0609764

[2] R. Stanley: Alternating permutations and symmetric functions, J. Combinatorial Theory Series A, Volume 114 (2007), pages 436-460.

[3] E. Steingrimsson, L. Williams, Permutation tableaux and permutation patterns, J. Combinatorial Theory Series A, Volume 114 (2007), pages 211-234. 